\title{
Pole Placement-Based NMPC of Hammerstein Systems and Its Application to Grade Transition Control of Polypropylene
}

\author{
He De-Feng and Yu Li \\ College of Information Engineering, Zhejiang University of Technology, Zhejiang, Hangzhou 310023, China \\ Correspondence should be addressed to He De-Feng, hdfzj@zjut.edu.cn
}

Received 1 July 2011; Accepted 11 September 2011

Academic Editor: Baocang Ding

Copyright ( $) 2012$ H. De-Feng and Y. Li. This is an open access article distributed under the Creative Commons Attribution License, which permits unrestricted use, distribution, and reproduction in any medium, provided the original work is properly cited.

\begin{abstract}
This paper presents a new nonlinear model predictive control (MPC) algorithm for Hammerstein systems subject to constraints on the state, input, and intermediate variable. Taking no account of constraints, a desired linear controller of the intermediate variable is obtained by applying pole placement to the linear subsystem. Then, actual control actions are determined in consideration of constraints by online solving a finite horizon optimal control problem, where only the first control is calculated and others are approximated to reduce the computational demand. Moreover, the asymptotic stability can be guaranteed in certain condition. Finally, the simulation example of the grade transition control of industrial polypropylene plants is used to demonstrate the effectiveness of the results proposed here.
\end{abstract}

\section{Introduction}

Hammerstein systems consist of the cascade connection of a static (memoryless) nonlinear function followed from a linear dynamic system. Under certain assumptions (such as fading memory), the Hammerstein model could approximately represent nonlinear dynamics of real systems and has been successfully applied to many kinds of industrial processes such as $\mathrm{pH}$ neutralization [1], distillation [2], and polymerization transition [3]. In recent years, constrained control of Hammerstein systems has become one of the most needed and yet very difficult tasks for the process industry.

Model predictive control (MPC) is an effective control algorithm for handling constrained control problems, and various MPC algorithms have been proposed for control of Hammerstein systems with constraints [4-11]. Making use of the geometry structure of Hammerstein model, [4-9] developed two-step MPC schemes for Hammerstein systems with input constraints, where the intermediate variable was firstly obtained by linear MPC and then the actual control was calculated by solving nonlinear algebraic equations, desaturation, and so forth. Although two-step MPC algorithms possess more light computational burden than that where the nonlinearities were incorporated into optimal control problems directly $[10,11]$, solving nonlinear algebraic equations will inevitably have error and the restricted control is generally different from the desired one for constrained Hammerstein systems [6]. Therefore, the performance and stability properties may be deteriorated in the presence of constraints. Moreover, to the best of authors' knowledge, the conversional two-step MPC schemes usually have limited ability to dealing with input constraints. So, [12] presented a new two-step MPC scheme for Hammerstein systems with overall constraints, where the actual controller was determined by solving a finite horizon optimization problem that was described as tracking quadratic, optimal trajectories of the intermediate variable. However, the online computational demand of optimization problem of the scheme is still an open question.

In this paper, we consider the problem of the grade transition control of industrial polypropylene plants and propose an efficient NMPC scheme for Hammerstein systems with overall constraints based on the pole placement method [13]. We firstly obtained desired trajectories of Hammerstein systems by using pole placement to the linear subsystem and then calculated actual control actions by solution of finite 
horizon optimal control problems. In order to reduce the online computational burden of the optimization problem, only the first control action is calculated and the remaining is approximated by solving nonlinear algebraic equations over predictive horizon. Thus, the performance and stability properties can be guaranteed in the face of overall constraints, with moderate increment of the computational demand. Finally, an example of the grade transition control of propylene plants is used to demonstrate the effectiveness of the proposed algorithm.

\section{Pole Placement-Based NMPC Algorithm}

Consider the discrete-time Hammerstein systems described by

$$
x(t+1)=A x(t)+B v(t), \quad v(t)=g(u(t), t), t=0,1, \ldots,
$$

where $x \in R^{n}, v \in R^{m}$, and $u \in R^{r}$ are the state, intermediate variable and control input, respectively. Vector field $g$ represents the nonlinear relationship between the input and intermediate variable, satisfying $g(0, t)=0$. It is assumed that $(A, B)$ is stabilizable and the state is available for feedback control. Without loss of generality, we also assume that the origin is the equilibrium of system (1).

Given constraints of system (1) as follows:

$$
\begin{aligned}
& x(t) \in M_{x}=\left\{x \in R^{n}: x^{\mathrm{LB}} \leq x \leq x^{\mathrm{UB}}\right\}, \quad t=0,1, \ldots, \\
& v(t) \in M_{v}=\left\{v \in R^{m}: v^{\mathrm{LB}} \leq v \leq v^{\mathrm{UB}}\right\}, \quad t=0,1, \ldots, \\
& u(t) \in M_{u}=\left\{u \in R^{r}: u^{\mathrm{LB}} \leq u \leq u^{\mathrm{UB}}\right\}, \quad t=0,1, \ldots,
\end{aligned}
$$

where sets $M_{x} \subseteq R^{n}, M_{v} \subseteq R^{m}$, and $M_{u} \subseteq R^{r}$ are constraints on the state, intermediate variable, and input, respectively. The goal of this paper is to design an efficient NMPC algorithm for the Hammerstein system (1) with the constraint (2).

2.1. Design of NMPC Algorithm. Consider $n$ desired poles, denoted by $\left\{\lambda_{1}, \ldots, \lambda_{n}\right\}$, of the linear subsystem of (1), which represent some desired performance of system (1). Associated with this set of desired poles is a linear controller of the intermediate variable

$$
v(t)=-K x(t)
$$

which is generated via Lyapunov's method without any constraints [13]. Since the controller (3) is unable to be implemented by real control action, here it is labeled as $v(t)^{d}=-K x(t)^{d}$ with associated closed-loop states $x(t)^{d}$.
Define a finite horizon objective function $J(t)$ as follows:

$$
\begin{gathered}
J(t)=\sum_{i=0}^{T p-1}\left\{\left[x(t+i+1 \mid t)-x(t+i+1)^{d}\right]^{\mathrm{T}}\right. \\
\quad \times Q\left[x(t+i+1 \mid t)-x(t+i+1)^{d}\right] \\
\left.+u(t+i \mid t)^{\mathrm{T}} R u(t+i \mid t)\right\},
\end{gathered}
$$

where the vector $\bullet(t+i \mid t)$ is $i$-step ahead prediction from time instant $t$, integer $0<T_{p}<\infty$ is the predictive horizon, and matrices $0 \leq Q$ and $0<R$ are the weighting matrices of the state and input, respectively. Then a new two-step MPC algorithm of Hammerstein systems with overall constraints is presented below.

Algorithm 1 (modified two-step MPC, MTMPC). This algorithm comprises the following steps:

Step 1. Given $n$ desired poles $\left\{\lambda_{1}, \ldots, \lambda_{n}\right\}$ and off-line compute the gain matrix $K$ in (3) such that the eigenvalues of $A-B K$ are those specified in vector $\left\{\lambda_{1}, \ldots, \lambda_{n}\right\}$.

Step 2. Set $T_{p}, Q, R$ in the objective function in (4).

Step 3. With $v(\cdot)^{d}=-K x(\cdot)^{d}$ and the current state $x(t)$, solve on-line the nonlinear algebra equations (5) without constraints to obtain $T_{p}$ desired control actions $u(t+i)^{d}$

$$
\begin{gathered}
v(t+i)^{d}-g\left(u(t+i)^{d}, t+i\right)=0, \\
x(t+i+1)^{d}=(A-B K) x(t+i)^{d}, \\
x(t)^{d}=x(t), \quad i=0,1, \ldots, T_{p}-1 .
\end{gathered}
$$

Step 4. With the current state $x(t)$, determine on-line the actual control action $u(t)$ as follows:

$$
\begin{aligned}
& u(t)=\arg \min _{u(t \mid t)} J(t), \\
& \text { s.t. } \quad x(t+i+1 \mid t)=A x(t+i \mid t)+B g(u(t+i \mid t), t+i), \\
& x(t+i+1)^{d}=(A-B K) x(t+i)^{d}, \\
& u(t+i \mid t)=u(t+i)^{d}, \quad i=1, \ldots, T_{p}-1, \\
& x(t+1 \mid t) \in M_{x}, \quad g(u(t \mid t), t) \in M_{v}, \quad u(t \mid t) \in M_{u}, \\
& x(t \mid t)=x(t)^{d}=x(t),
\end{aligned}
$$

where $J(t)$ is defined by (4).

Step 5. Implement $u(t)$, set $t=t+1$, and go back Step 3 .

Remark 2. For the algorithm here, only the first control action (i.e., $u(t \mid t)$ ) is optimized subject to constraints at each time. So, the online optimization problem has only $r$ decision variables versus $T_{p} r$ decision variables for general 
nonlinear MPC, which results in a significant reduction of the online computational demand since the computational demand, in general, grows exponentially with the number of decision variables [14]. Thus, the predictive horizon $T_{p}$ should be chosen reasonably large to ensure adequate performance. On the other hand, since only the first control is implemented in MPC scheme, it is expected that only enforcing constraints for the first prediction should give excellent results. In addition, the closed-loop performance of MPC systems clearly depends on how $n$ desired poles $\left\{\lambda_{1}, \ldots, \lambda_{n}\right\}$ are arranged. The further work will focus on how to determine $\left\{\lambda_{1}, \ldots, \lambda_{n}\right\}$ optimally.

It should be pointed out that the feasibility of optimization problem (6) may not be guaranteed theoretically with respect to arbitrary constraints in (2). However, it is usually ensured in practice via adjusting the intermediate variable constraint. Next, we show that the closed-loop MPC system (1) is asymptotically stable, provided that the feasibility of (6) is hold.

2.2. Analysis of Stability. In terms of Algorithm MTMPC, the MPC law of constrained Hammerstein system (1), (2) is defined as

$$
u(t)^{\mathrm{MPC}}=u(t \mid t), \quad t=0,1, \ldots
$$

with the closed-loop system

$$
\begin{aligned}
x(t+1) & =A x(t)+B g\left(u(t)^{\mathrm{MPC}}, t\right) \\
& =(A-B K) x(t)+B\left[K x(t)+g\left(u(t)^{\mathrm{MPC}}, t\right)\right] .
\end{aligned}
$$

Definition 3. Set $M \subseteq R^{n}$ is called an attractive region of system (8) if for all $x(t) \in M$, the system trajectories $x(s ; x(t)) \rightarrow 0$ when $s \rightarrow+\infty$ and satisfies

$x(t) \in M \Longrightarrow x(t+1) \in M, \quad \forall u(t)^{\mathrm{MPC}} \in M_{u v}, t=0,1, \ldots$,

where subset $M_{u v}=\left\{u \in R^{r}: g(u, \cdot) \in M_{v}\right\} \subseteq M_{u}$.

Let a set of points $\left\{\lambda_{1}, \ldots, \lambda_{n}\right\}$ be the desired poles of the linear subsystem $x(t+1)=(A-B K) x(t)$. Then from inverse Lyapunov's theorem [13], there is systematic matrices $P>0$ and $Q>0$ such that

$$
(A-B K)^{\mathrm{T}} P(A-B K)-P<-Q .
$$

In following, we present the result on our proposed algorithm.

Theorem 4. Consider the system (1), (2), there exists a nonempty region $M$ such that the closed-loop system (8) is asymptotically stable if the inequality (11) is satisfied

$$
V(x(t+1)) \leq V\left(x(t+1)^{d}\right), \quad \forall x(t) \in M, t=0,1, \ldots,
$$

where function $V(x)=x^{\mathrm{T}} P x$ and $x(t+1)^{d}=(A-B K) x(t)^{d}$ with $x(t)^{d}=x(t)$. Moreover, the set $M$ is an attractive region of system (8).

Proof. Consider the system (1), (2), define a level set $M$ of function $V(x)$ as

$$
M=\left\{x \in R^{n}: V(x) \leq c, c>0\right\} \subseteq M_{x}
$$

such that $u(t)^{\mathrm{MPC}} \in M_{u v}$, for all $x(t) \in M, t=0,1, \ldots$. Note that the set $M$ is always nonempty since the constraint in (2) includes the origin as the internal point.

Let function $V(x)$ be a Lyapunov function candidate of system (8). Then, for any $x(t) \in M$, we have

$$
\begin{aligned}
& V(x(t+1))-V(x(t)) \\
&=x(t+1)^{\mathrm{T}} P x(t+1)-x(t)^{\mathrm{T}} P x(t) \\
&=x(t)^{\mathrm{T}}(A-B K)^{\mathrm{T}} P(A-B K) x(t)^{\mathrm{T}} \\
& \quad-x(t)^{\mathrm{T}} P x(t)+2 x(t)^{\mathrm{T}}(A-B K)^{\mathrm{T}} P B \Delta+\Delta^{\mathrm{T}} B^{\mathrm{T}} P B \Delta \\
&= x(t)^{\mathrm{T}}\left[(A-B K)^{\mathrm{T}} P(A-B K)-P\right] x(t) \\
&+2 x(t)^{\mathrm{T}}(A-B K)^{\mathrm{T}} P B \Delta+\Delta^{\mathrm{T}} B^{\mathrm{T}} P B \Delta,
\end{aligned}
$$

where $\Delta=g\left(u(t)^{\mathrm{MPC}}, t\right)+K x(t)=g\left(u(t)^{\mathrm{MPC}}, t\right)-v(t)^{d}$. Substituting (10) into (13) yields

$$
\begin{aligned}
& V(x(t+1))-V(x(t)) \\
&=-x(t)^{\mathrm{T}} Q x(t)+2[(A-B K) x(t)]^{\mathrm{T}} P B \Delta+\Delta^{\mathrm{T}} B^{\mathrm{T}} P B \Delta \\
&=-x(t)^{\mathrm{T}} Q x(t)+2 x(t)^{\mathrm{T}} A^{\mathrm{T}} P B \Delta \\
&+2(g-\Delta)^{\mathrm{T}} B^{\mathrm{T}} P B \Delta+\Delta^{\mathrm{T}} B^{\mathrm{T}} P B \Delta \\
&=-x(t)^{\mathrm{T}} \mathrm{Q} x(t)+2 x(t+1)^{\mathrm{T}} P B \Delta-\Delta^{\mathrm{T}} B^{\mathrm{T}} P B \Delta \\
&=-x(t)^{\mathrm{T}} \mathrm{Q} x(t)-x(t+1)^{d \mathrm{~T}} P x(t+1)^{d} \\
&+x(t+1)^{\mathrm{T}} P x(t+1) \\
&=-x(t)^{\mathrm{T}} Q x(t)-V\left(x(t+1)^{d}\right)+V(x(t+1)) .
\end{aligned}
$$

From the inequality (11), it is straight forward to obtain that

$$
V(x(t+1))-V(x(t)) \leq-x(t)^{\mathrm{T}} Q x(t), \quad \forall x(t) \in M .
$$

Hence, function $V(x)$ is a Lyapunov function of system (8), and the system is asymptotically stable in region $M$. Furthermore, from the definition of attractive regions and set $M$ in (12), we derive that $M$ is an attractive region of system (8).

This establishes the theorem. 
Remark 5. From the proof of the above theorem, we know that the stability property of closed-loop system (8) is guaranteed by the linear controller of the intermediate variable, provided that the feasibility of optimization problem (6) and condition (11) holds. This suggests that the stability and optimality can be separated to some extent. Therefore, the design parameter of the objective function in (4) can be tuned freely to achieve more satisfactory performance, regardless the stability of the closed-loop system. In addition, it is pointed out that the attractive region $M$ obtained here is merely a theoretical concept and the computation of $M$ is not an easy task.

Remark 6. Instead of conventional two-step MPC algorithms, where the actual control was determined by solving nonlinear algebraic equations based on desired controllers of the intermediate variable resulted from linear MPC, actual control actions here are derived by solving a finite horizon optimal control problem (i.e., nonlinear MPC), which is defined as tracking the desired state trajectories driven by pole placement state feedback controller. Thus, overall constraints of Hammerstein systems are taken into account when designing the predictive controller, and then performance and stability properties are guaranteed. Meanwhile, the ability to handling constraints is enhanced in the algorithm proposed here. Note that the linear controller of intermediate variables in (3) can be any one that stabilizes the linear subsystems of Hammerstein models with certain desired performance.

\section{Simulation Example}

Taking an example of polypropylene (PP) grade transition control, we illustrate the effectiveness of the proposed algorithm.

In propylene polymerization industry, melt index (MI, $\mathrm{g} / 10 \mathrm{~min}$ ) is usually used to identify grade indices of PP homopolymer and both of MI and concentration of ethylene $\left(E_{t}, \%\right)$ in PP denote grade indices of total phase PP $[12,15-$ $18]$. This implies that the nature of grade transition process is to operate the response of the variables $\mathrm{MI}$ and $E_{t}$ in polymer. Then according to the propylene polymerization mechanism, we have the following mathematical model which formulates the dynamics of the grade transition in polypropylene process [12]:

$$
\begin{gathered}
{\left[\begin{array}{l}
\dot{x}_{1} \\
\dot{x}_{2}
\end{array}\right]=\left[\begin{array}{cc}
-\frac{1}{\tau} & 0 \\
0 & -\frac{1}{\tau}
\end{array}\right]\left[\begin{array}{l}
x_{1} \\
x_{2}
\end{array}\right]+\left[\begin{array}{cc}
\frac{1}{\tau} & 0 \\
0 & \frac{1}{\tau}
\end{array}\right]\left[\begin{array}{l}
g_{1}(u, t) \\
g_{2}(u, t)
\end{array}\right],} \\
{\left[\begin{array}{l}
g_{1}(u, t) \\
g_{2}(u, t)
\end{array}\right]=\left[\begin{array}{c}
k_{1}+\frac{k_{2}}{u_{1}}+k_{3} \log \left(k_{4}+k_{5} u_{2}+k_{6} u_{3}\right) \\
\frac{k_{7} u_{2}+1}{k_{8} / u_{3}+k_{9} u_{3}+k_{10}}
\end{array}\right],} \\
y(t)=\left[\begin{array}{ll}
1 & 0 \\
0 & 1
\end{array}\right] x(t), \quad t \geq 0,
\end{gathered}
$$

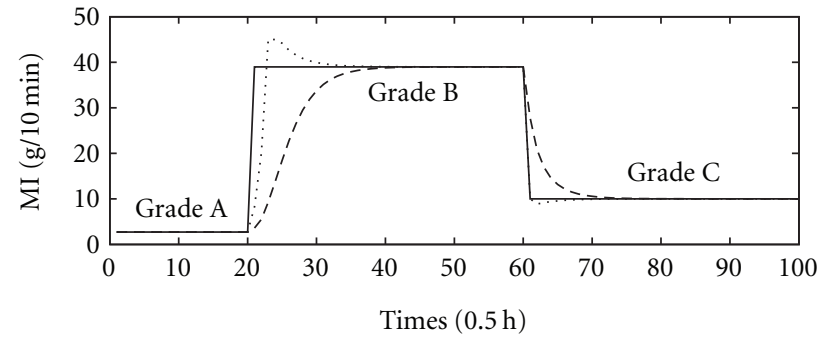

(a) Melt index

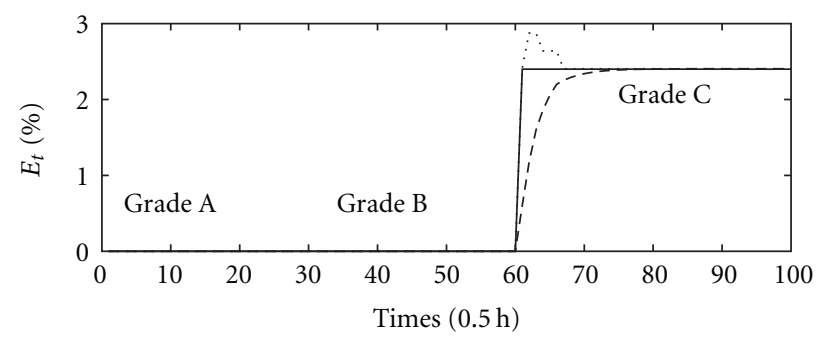

(b) Concentration of ethylene in polypropylene

Figure 1: MI and $E_{t}$ output trajectories of grade transition processes. (Solid line is the set-point, dotted line the instantaneous, and dashed line the cumulative).

where states $x_{1}$ and $x_{2}$ are the cumulative $\mathrm{MI}\left(\mathrm{MI}_{c}, \mathrm{~g} / 10 \mathrm{~min}\right)$ and $E_{t}\left(E_{t c}, \%\right)$ of $\mathrm{PP}$, respectively; intermediate variables $g_{1}$ and $g_{2}$ denote the instantaneous $\mathrm{MI}\left(\mathrm{MI}_{i}, \mathrm{~g} / 10 \mathrm{~min}\right)$ and $E_{t}\left(E_{t i}, \%\right)$ of $\mathrm{PP}$, respectively; input, $u_{1}, u_{2}, u_{3}$ denote the reaction temperature $T(K)$, concentration ratio of hydrogen to propylene $C_{\mathrm{H} 2} / C_{\mathrm{m}}(\%)$, and concentration ratio of ethylene to propylene $C_{\mathrm{m} 2} / C_{\mathrm{m}}(\%)$, respectively, and parameter $\tau$ is the average polymer residence time $(\tau=2 \mathrm{~h})$. The parameters of model $k_{i}(i=1, \ldots, 10)$ can be identified on-line by industrial data [15].

The grade transition sequence considered here is $\mathrm{A} \rightarrow$ $\mathrm{B} \rightarrow \mathrm{C}$, where grades $\mathrm{A}$ and $\mathrm{B}$ are $\mathrm{PP}$ homopolymer and $\mathrm{C}$ is total phase PP. It should be pointed out that the feed flow rate of ethylene is always zero (i.e., $\left[C_{\mathrm{m} 2} / C_{\mathrm{m}}\right]=0$ ) in production of PP homopolymer grades $\mathrm{A}$ and $\mathrm{B}$. The grade transition process is defined by the set-point of states and input/output constraints as shown in Table 1, where superscripts $c, i$, and $\Delta$ denote accumulation, instantaneous value, and increment constraints, respectively. Each grade transition is optimized over $10 \mathrm{~h}$ horizon with a sampling interval of $0.5 \mathrm{~h}$.

In the simulation running, the closed-loop desired poles of system (16) are placed at $\left\{\lambda_{1}, \lambda_{2}\right\}=\{0.72,0.60\}$, and the corresponding state-feedback matrix $K$ in (3) is computed by $K=[0.12,0 ; 0,0.6]$. Moreover, the parameters in the objective function (4) are chosen as penalty on the states, $Q=q I_{2}$, with $q=1$, penalty on the inputs, $R=I_{3}$ and a horizon length of $T_{p}=20$. Assume that grade transition $\mathrm{A} \rightarrow \mathrm{B}$ takes place at the tenth hour and $\mathrm{B} \rightarrow \mathrm{C}$ at the thirtieth hour. Then some curves of the grade transition process being controlled by NMPC law are shown in Figures 1 and 2, where the solid line in Figure 1 is the set-point, the dotted line the instantaneous, and the dashed line the cumulative. 
TABLE 1: Grade indices and transition constraints.

\begin{tabular}{lccccc}
\hline Grade & MI $(\mathrm{g} / 10 \mathrm{~min})$ & $E_{t}(\%)$ & $T(\mathrm{~K})$ & $C_{\mathrm{H} 2}(\%)$ & $C_{\mathrm{m} 2} / C_{\mathrm{m}}(\%)$ \\
\hline $\mathrm{A}$ & 2.7 & - & 343.15 & 0.050 & - \\
$\mathrm{B}$ & 39.0 & - & 343.15 & 0.330 & - \\
$\mathrm{C}$ & 10.0 & 2.4 & 343.15 & 0.236 & 1.99 \\
$\mathrm{~A} \rightarrow \mathrm{B}$ & {$[2.4,42.0]^{c}$} & - & {$[341.15,345.15]$} & {$[0.02,0.35]$} & - \\
& {$[2.0,45.0]^{i}$} & - & - & {$[-0.1,0.10]^{\Delta}$} & - \\
$\mathrm{B} \rightarrow \mathrm{C}$ & {$[8.0,42.0]^{\mathrm{c}}$} & {$[0.0,2.5]^{\mathrm{c}}$} & {$[341.15,345.15]$} & {$[0.20,0.35]$} & {$[0.00,2.50]$} \\
& {$[6.0,45.0]^{i}$} & {$[0.0,2.6]^{i}$} & - & {$[-0.1,0.10]^{\Delta}$} & {$[-0.1,0.10]^{\Delta}$} \\
\hline
\end{tabular}

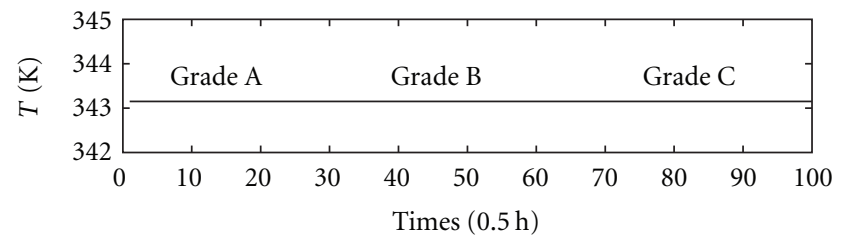

(a) Reaction temperature

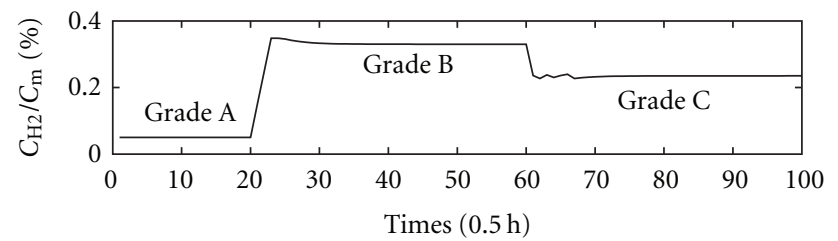

(b) Concentration ratio of hydrogen to propylene

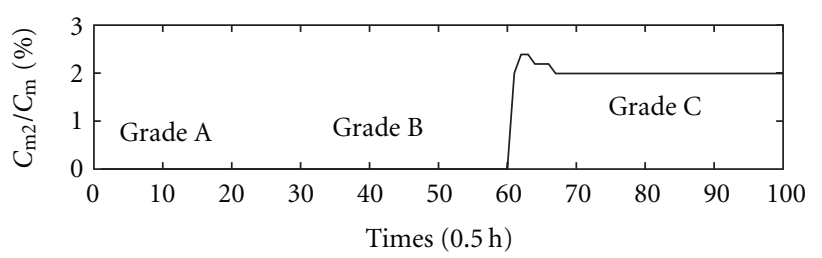

(c) Concentration ratio of ethylene to propylene

FIGURE 2: Input profiles of grade transition processes.

In order to calculate the quantity of off-specification polymer in grade transition process, we employ the criterion where on-specification polymer is defined as the polymer with $\mathrm{MI}_{c}$ and $E_{t c}$ within a $\pm 5 \%$ deviation of desired specification in Table 1. All other polymers beyond the criterion are categorized as off-specification production. Similarly, the transition time is defined as the time interval when the cumulative properties of polymer are out of the specification range $\pm 5 \%$ of the previous grade and lasting until the cumulative properties enter and stay within the specification range $\pm 5 \%$ of the new target grade. Therefore, the time of grade $\mathrm{A} \rightarrow \mathrm{B}$ transition is 8.5 hours and the time of grade $\mathrm{B} \rightarrow \mathrm{C}$ transition is 6.5 hours. Compare to the time of 10 hours in actual grade transition operation, this decreases the quantity of off-specification polymer in grade transition process and then increases the productive time of on-specification polymer. Also, Figure 2(a) suggests that there is no change in reaction temperature during the grade transition processes, which is desired in actual operation. Finally, it can be seen that the control laws, intermediate variables, and the states in the grade transition operation do not violate the constraints in Table 1.

\section{Conclusion}

Together with the pole placement method, the paper presented an efficient MPC algorithm for control of Hammerstein systems subject to constraints on states, inputs, and intermediate variables. Applying pole placement to the linear subsystem of Hammerstein models yielded linear controllers of the intermediate variable and then the actual control action was determined by online solving an optimization problem. The size of the online optimization problem depended only on the number of inputs, not on the predictive horizon, which greatly reduced the computational demand of the algorithm. With condition of feasibility of the optimization problem, the asymptotical stability of the closed-loop system with constraints was guaranteed and the results on simulation example of grade transition control of polypropylene plants showed the effectiveness of the results obtained here.

\section{Acknowledgments}

This work is supported by the National Natural Science Foundation of China under Grant no. 60904040, Specialized Research Fund for Doctoral Program of Higher Education of China under Grant no. 20093317120002 and Natural Science Foundation of Zhejiang Province under Grant no. Y1100911. Finally, the authors are grateful to the reviews' constructive comments. This paper includes revised and extended text of the first author's presentation at the 29th CCC, July 27, 2010, Beijiang, China.

\section{References}

[1] Z. Hasiewicz, "Hammerstein system identification by the Haar multiresolution approximation," International Journal of Adaptive Control and Signal Processing, vol. 49, pp. 691-717, 1999.

[2] N. Bhandari and D. Rollins, "Continuous-time Hammerstein nonlinear modeling applied to distillation," AIChE Journal, vol. 50, no. 2, pp. 530-533, 2004.

[3] D. F. He, L. Yu, and M. S. Xue, "On operation guidance for grade transition in propylene polymerization plants," in Proceedings of the 19th Chinese Process Control Conference, pp. 339-342, Beijiang, China, 2008, (Chinese). 
[4] Q. M. Zhu, K. Warwick, and J. L. Douce, "Adaptive general predictive controller for nonlinear systems," IEE Proceedings D, vol. 138, no. 1, pp. 33-40, 1991.

[5] K. P. Fruzzetti, A. Palazoğlu, and K. A. McDonald, "Nonlinear model predictive control using Hammerstein models," Journal of Process Control, vol. 7, no. 1, pp. 31-41, 1997.

[6] B. C. Ding, Y. G. Xi, and S. Y. Li, "Stability analysis on predictive control of discrete-time systems with input nonlinearity," Acta Automatica Sinica, vol. 29, no. 6, pp. 827-834, 2003.

[7] B. C. Ding, Y. G. Xi, and S. Y. Li, "On the stability of output feedback predictive control for systems with input nonlinearity," Asian Journal of Control, vol. 6, no. 3, pp. 388397, 2004.

[8] B. Ding and Y. Xi, "A two-step predictive control design for input saturated Hammerstein systems," International Journal of Robust and Nonlinear Control, vol. 16, no. 7, pp. 353-367, 2006.

[9] H. T. Zhang, H. X. Li, and G. Chen, "Dual-mode predictive control algorithm for constrained Hammerstein systems," International Journal of Control, vol. 81, no. 10, pp. 1609-1625, 2008.

[10] H. H. J. Bloemen and T. J. J. van den Boom, "Model-based predictive control for Hammerstein systems," in Proceedings of the 39th IEEE Conference on Decision and Control, vol. 5, pp. 4963-4968, Sydney, Australia, 2000.

[11] H. H. J. Bloemen, T. J. J. van den Boom, and H. B. Verbruggen, "Model-based predictive control for Hammerstein-Wiener systems," International Journal of Control, vol. 74, pp. 482-495, 2005.

[12] D. F. He and L. Yu, "Nonlinear predictive control of constrained Hammerstein systems and its research on simulation of polypropylene grade transition," Acta Automatica Sinica, vol. 35, no. 12, pp. 1558-1563, 2009 (Chinese).

[13] C. T. Chen, Linear system Theory and Design, Oxford University Press, New York, NY, USA, 3rd edition, 1999.

[14] A. Zheng, "A computationally efficient nonlinear MPC algorithm," in Proceedings of the American Control Conference, pp. 1623-1627, Albuquerque, NM, USA, June 1997.

[15] J. R. Richards and J. P. Congalidis, "Measurement and control of polymerization reactors," Computers and Chemical Engineering, vol. 30, no. 10-12, pp. 1447-1463, 2006.

[16] H. S. Yi, J. H. Kim, C. Han, J. Lee, and S. S. Na, "Plantwide optimal grade transition for an industrial high-density polyethylene plant," Industrial and Engineering Chemistry Research, vol. 42, no. 1, pp. 91-98, 2003.

[17] Y. Wang, H. Seki, S. Ohyama, K. Akamatsu, M. Ogawa, and M. Ohshima, "Optimal grade transition control for polymerization reactors," Computers and Chemical Engineering, vol. 24, no. 2-7, pp. 1555-1561, 2000.

[18] T. Kreft and W. F. Reed, "Predictive control of average composition and molecular weight distributions in semibatch free radical copolymerization reactions," Macromolecules, vol. 42, no. 15, pp. 5558-5565, 2009. 

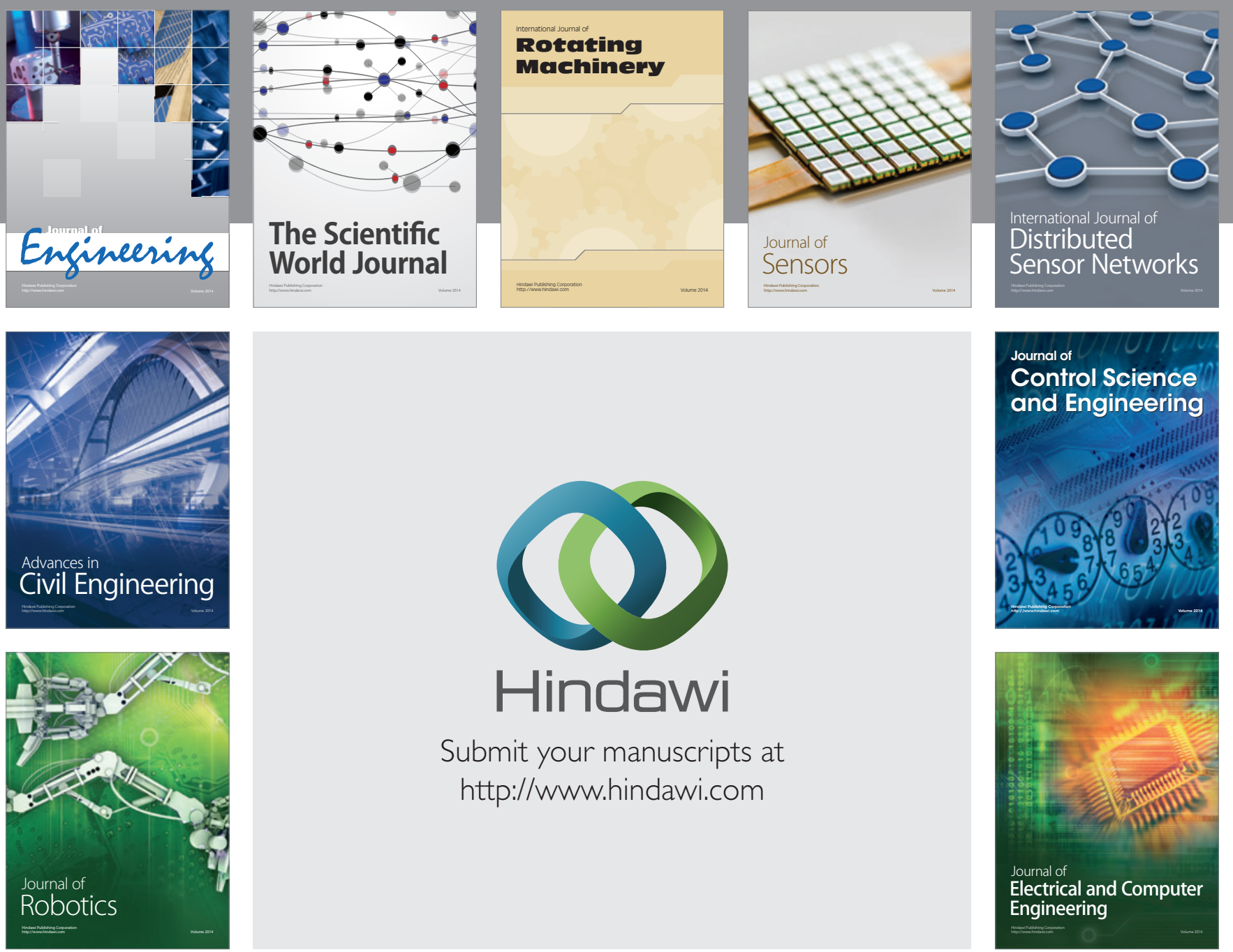

Submit your manuscripts at

http://www.hindawi.com
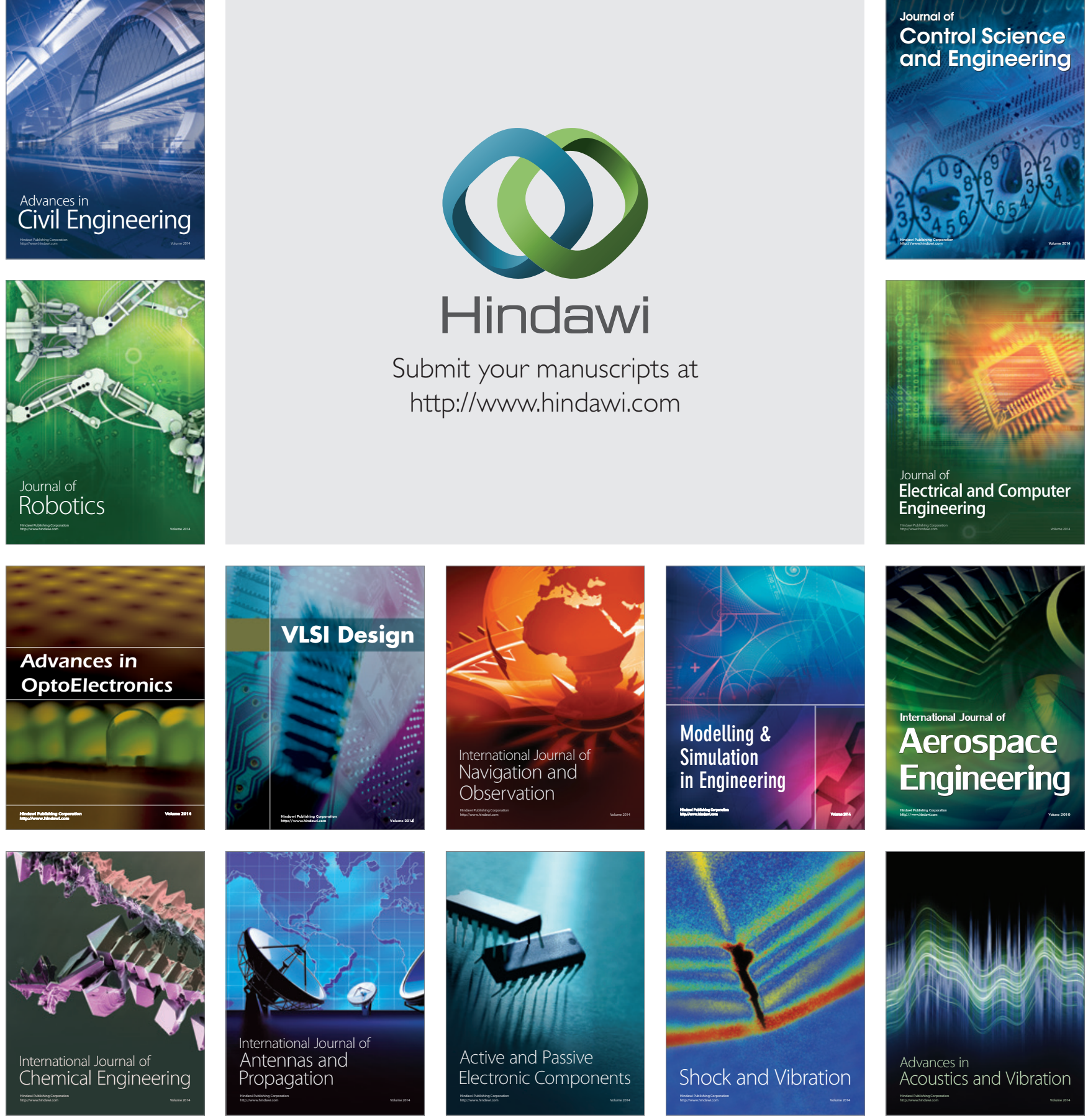